\title{
Spectroscopic monitoring of the Herbig Ae star HD 104237 ${ }^{\star}$
}

\section{Non-radial pulsations, mode analysis, and fundamental stellar parameters}

\author{
A. Fumel ${ }^{1,2}$ and T. Böhm ${ }^{1,2}$ \\ 1 Université de Toulouse, UPS-OMP, IRAP, Toulouse, France \\ e-mail: torsten . boehm@irap.omp.eu \\ 2 CNRS, IRAP, 14, avenue Edouard Belin, 31400 Toulouse, France
}

Received 3 August 2011 / Accepted 8 March 2012

\begin{abstract}
Context. Herbig Ae/Be stars are intermediate-mass pre-main sequence (PMS) stars showing signs of intense activity and strong stellar winds, whose origin is not yet understood in the frame of current theoretical models of stellar evolution for young stars. In addition, the evolutionary tracks of the earlier Herbig Ae stars cross the theoretical PMS instability strip located roughly in the same area of the HR diagram as the $\delta$ Scuti variables. Many of these stars exhibit pulsations of $\delta$ Scuti type.

Aims. Understanding the internal structure of pulsating Herbig Ae stars based on asteroseismic studies will help constraining the origin of their tremendous activity, winds, and variability. It is therefore necessary to investigate the location of the PMS instability strip and of its boundaries, and to extend the sample of observed and studied pulsating Herbig Ae stars. The aim of this work was to carry out a thorough analysis of the line profile variations of the prototype Herbig Ae star HD 104237 based on high-resolution spectroscopy and to redetermine precisely its fundamental parameters, which are the basic ingredients of a forthcoming asteroseismic modeling.

Methods. HD 104237 is a pulsating Herbig Ae star with eight detected frequencies based on the analysis of radial velocity variations. In this article, we reinvestigated an extensive high-resolution quasi-continuous spectroscopic data set in order to search for very faint indications of non-radial pulsations in the line profile by working on dynamical spectra of equivalent photospheric (LSD) profiles of HD 104237. A 2D Fourier analysis (F2D) was performed of the entire profile and the temporal variation of the central depth of the line was studied with the time-series analysis tools Period04 and SigSpec. We present the results of these analysis including the mode identification corresponding to the detected dominant frequency, as well as a new determination of its fundamental stellar parameters. Results. The analysis of spectroscopic data set of April 22-25 obtained at SAAO in 1999 has confirmed the presence of multiple oscillation modes of low-degree $\ell$ in HD 104237 and led to the first direct detection of a non-radial pulsation mode in this star: the dominant mode F1 was identified by the Fourier 2D method having a degree $\ell$ value comprised between 1 and 2 , the symmetry of the pattern variation indicating an azimuthal order of \pm 1 . The detailed study of the fundamental stellar parameters has provided a $T_{\text {eff }}, \log$ $\mathrm{g}$, and iron abundance of $8550 \pm 150 \mathrm{~K}, 3.9 \pm 0.3$, and $-4.38 \pm 0.19$ (i.e. $[\mathrm{Fe} / \mathrm{H}]=+0.16 \pm 0.19$ ), respectively.
\end{abstract}

Key words. stars: pre-main-sequence - stars: oscillations - stars: individual: HD 104237 - binaries: spectroscopic stars: fundamental parameters

\section{Introduction}

Pre-main sequence (PMS) stars of intermediate mass (2 to $10 M_{\odot}$ ) are known as Herbig $\mathrm{Ae} / \mathrm{Be}$ (hereafter $\mathrm{HAeBe}$ ) stars, following a first classification by Herbig (1960); for a review, see Waters \& Waelkens (1998). Their spectra reveal strong infrared excesses indicative of a dense circumstellar environment. Intense signs of stellar activity, variability, and winds are observed. Indeed, the frequently observed emission in $\mathrm{Mg}$ II $\mathrm{h}$ and k lines, Ca II IR triplet, Ca II H and K line, He I $5876 \AA$, many Fe II lines, Na I D, N V, Si IV and C IV resonance lines, imply the high temperature of a chromosphere or corona to be formed. Short-term variability of many of these lines are observed and P Cygni profiles of $\mathrm{H} \alpha, \mathrm{H} \beta, \mathrm{Mg}$ II $\mathrm{h}$ and $\mathrm{k}$ lines present in the spectra of some of these stars indicate the presence of strong and structured stellar winds (see e.g. Praderie et al. 1982, 1986; Catala \& Talavera 1984; Catala et al. 1986a,b; Catala \& Kunasz 1987; Catala 1988; Böhm \& Catala 1995; Böhm et al. 1996).

\footnotetext{
^ Based on observations collected at the $1.9 \mathrm{~m}$ SAAO telescope.
}

A wide-spread idea consists of invoking magnetism as being responsible of these active stellar phenomena, requiring either the presence of a primordial magnetic field or a dynamo mechanism whose basic ingredients are classically convection and rotation. On one hand, recent spectropolarimetric observations of Herbig stars indicate the presence of significant magnetic fields only in a small $10 \%$ fraction of them (Wade et al. 2007), these results agree with the primordial fossil field hypothesis. On the other hand, considering a possible dynamo mechanism, the position of HAeBe stars in the HR diagram indicates that they are in the radiative phase of their quasi-static contraction toward the main sequence (Iben 1965; Gilliland 1986; Palla $\&$ Stahler 1993). Therefore, in absence of subphotospheric convection, a classical solar-type magnetic dynamo mechanism cannot be at work and the observed activity remains paradoxical. While an external origin, involving an accretion disk as invoked for explaining the activity in classical TTauri stars (Bertout et al. 1988), cannot be ruled out, growing evidence indicates that the energy needed to produce this activity is of internal stellar origin (Böhm \& Catala 1995); theoretical explanations might invoke 
deuterium shell burning (Palla \& Stahler 1990) or an alternative dynamo mecanism based on a non-convective turbulent layer (Lignieres et al. 1996). It is a major concern for testing young stellar evolutionary theory to solve this still-open question about HAeBe stellar activity by constraining the internal structure of these objects using asteroseismic techniques, i.e. the analysis and modeling of stellar pulsations, if observed.

The existence of these stellar pulsations in HAeBe stars are known since Breger (1972) discovered two pulsating candidates, V588 Mon and V589 Mon, in the young open cluster NGC 2264. Subsequently, two additional pulsating Herbig stars were detected: HR 5999 (Kurtz \& Marang 1995) and HD 104237 (Donati et al. 1997). Motivated by these detections, Marconi \& Palla (1998) investigated theoretically a PMS instability strip for the first three radial modes of pulsations and concluded on a topology of this instability strip, which corresponded roughly to the same area in the HR diagram as the $\delta$ Scuti variables. The study of Marconi \& Palla (1998) showed that most of the Herbig Ae stars are expected to cross it for a significant fraction of their evolution to the main sequence (5 to $10 \%$ of their PMS phase). Studying the largest number of PMS pulsating stars will allow us to constrain the boundaries of the PSM instability strip and to caracterize it. The priority should be given to multiperidodic pulsators, which are the most promising candidates for an asteroseismic modeling. As of today, a significant number of Herbig Ae stars, either field stars or members of young open clusters, have indeed revealed to be pulsating at timescales typical of $\delta$ Scuti stars, i.e. with short periods (from $\approx 20 \mathrm{~min}$ to several hours) and low amplitudes (from mmag to few hundreths of mag in case of photometry, and less than typically 1$2 \mathrm{~km} \mathrm{~s}^{-1}$ for radial velocity studies) (for reviews see e.g. Catala 2003; Marconi \& Palla 2004; Zwintz et al. 2004; Zwintz 2008). Most of them have been studied in photometry, but only very few in spectroscopy (e.g. Böhm et al. 2004, 2009). However, owing to cancellation effects, only modes of low degree are detected in photometry or radial velocity curves. For a deeper analysis high-resolution spectroscopy is necessary to study the weak variations induced by non-radial pulsations in the rotationallybroadened photospheric line profiles. It should be noted that binarity is common among HAeBe stars with approximately 60\% (Baines et al. 2006). HD 104237 is also a spectroscopic binary, which tremendously complicates the analysis of its spectrum.

This article is the second of a series on the prototype Herbig Ae star HD 104237, starting with the article by Böhm et al. (2004). The first article presented the detection of multiperiodic oscillations in the radial velocity curves of the star and the analysis of its binary orbit, among others. In this present article, we describe the direct search and detection of oscillations in the line profiles, the mode identification of the dominant non-radial pulsation mode, and a fundamental stellar parameter determination by spectroscopic means. This work therefore represents the basis for the asteroseismic modeling we will present in a forthcoming article (Fumel et al., in prep.).

The article is structured as follows: in Sect. 2, we review in detail previous works on HD 104237, Sect. 3 sums up the spectroscopic observations we worked with and describes the data reduction. Section 4 presents our detection and identification of non-radial pulsations in HD 104237, Sect. 5 describes the spectral continuum determination tool we developed to obtain optimized high-quality spectra. In Sect. 6 we detail the fundamental parameter determination of HD 104237 we carried out, and finally we conclude and discuss our results in Sect. 7.

\section{Previous works on HD 104237}

$\mathrm{Hu}$ et al. (1989, 1991) derived from photometric and spectroscopic studies that HD 104237, or DX Cha, is a member of the Herbig Ae/Be group. Moreover, it is a prototype of this group of stars and a particularly suitable target because it is very bright $\left(m_{\mathrm{v}}=6.6\right)$, which allows monitoring with short exposure times in high-resolution spectroscopic mode. HD 104237 is a multiple system whose primary component (hereafter simply called HD 104237 or the "primary") is a Herbig Ae star with several low-mass companions at separations between 1 " and $15^{\prime \prime}$ (Feigelson et al. 2003), including a close K3 companion in an eccentric 19.9-day orbit (hereafter HD 104237b or the "secondary"). This very close component of approximate spectral class K3 forms a spectroscopic binary with the primary (Böhm et al. 2004). Indeed, K3-type spectral features (including Li I $6707 \AA$ and Ca I $6718 \AA$ lines) are observed in the composite spectra (Feigelson et al. 2003). As a consequence, the pollution by the secondary spectrum has to be taken into account in a precise fundamental parameter analysis.

\subsection{Previous fundamental parameter determination}

The strong IR excesses and the UV-extinction caused by the presence of CS dust, the emission components in many absorption lines, the P Cygni profiles observed notably in the Balmer lines, and the spectroscopic binary pollution make the determination of most fundamental stellar parameters of HD 104237 difficult. Thus, the spectral types available in the literature for HD 104237 range from A0 to A8 (implying a broad range in possible $T_{\text {eff }}$ values) and its bolometric luminosity from $\approx 20$ to $60 L_{\odot}$. The most important parameters of HD 104237 (primary and secondary) found in the literature are chronologically summarized in Table 1.

Using the accurate absolute astrometric and photometric data yielded by the Hipparcos satellite, van den Ancker et al. (1997) computed new $T_{\text {eff }}, \log \left(L_{\star} / L_{\odot}\right)$, mass and age values for HD 104237, which were refined in van den Ancker et al. (1998). They should provide, as of today, the most reliable parameter determination. The uncertainties on the effective temperature (see van den Ancker et al. 1997) were estimated to correspond typically to one subclass in spectral type and were not determined in a statistical procedure.

Using evolutionary tracks and isochrones from Palla \& Stahler (2001) and stellar parameters from van den Ancker et al. (1998) and Grady et al. (2004), Böhm et al. (2004) concluded on an age of 2 Myr for HD 104237, in agreement with van den Ancker et al. (1997, 1998) and Feigelson et al. (2003), Böhm and collaborators independently concluded on a value of the stellar mass $M_{\star}=2.2 \pm 0.1 M_{\odot}$, close to previous results. In addition, they calculated a mass ratio $M_{\mathrm{P}} / M_{\mathrm{S}}$ of $1.29 \pm 0.02$, based on measures of the primary and secondary radial velocities with respect to the binary systemic velocity, which enabled them to estimate the mass of the secondary, $M_{\mathrm{sec}}=1.7 \pm 0.1 M_{\odot}$. Assuming the same age for both components, they confirmed a K3 spectral type for the secondary and assessed a temperature of about $4750 \mathrm{~K}$ and a luminosity of one tenth of $L_{\mathrm{P}}$.

Acke \& Waelkens (2004) used the $T_{\text {eff }}$ and $\log g$ presented in Meeus et al. (2001, and references therein) to carry out a chemical analysis of several PMS stars including HD 104237. They measured the equivalent widths of many chemical elements and converted them into abundances using the program MOOG (Sneden 1973), but taking into account neither the binarity of this star (which adds a continuum veiling along with 
A. Fumel and T. Böhm: Spectroscopic monitoring of the Herbig Ae star HD 104237. II.

Table 1. Stellar parameters of HD 104237.

\begin{tabular}{|c|c|c|c|c|c|c|c|c|c|c|c|}
\hline Ref. & & Sp. type & $\log T_{\text {eff }}$ & $T_{\text {eff }}(\mathrm{K})$ & $\log \left(L_{\star} / L_{\odot}\right)$ & $\log g$ & $M_{\star} / M_{\odot}$ & $R_{\star} / R_{\odot}$ & Age (Myr) & $v \sin i\left(\mathrm{~km} \mathrm{~s}^{-1}\right)$ & $i\left(^{\circ}\right)$ \\
\hline [1] & $\mathrm{P}$ & A0Vpe & & & & & & & & & \\
\hline [2] & $\mathrm{P}$ & $\mathrm{A} 4 \mathrm{Ve}$ & 3.93 & 8450 & 1.34 & 4.1 & $2.1^{a}$ & 2.2 & & & \\
\hline [3] & $\mathrm{P}$ & A7IVe & & & & & & & & & \\
\hline [4] & $\mathrm{P}$ & A0Vpe [1] & $3.98 \pm 0.05$ & $9550^{ \pm 550}$ & $1.77 \pm 0.06$ & 3.9 & $2.5 \pm 0.1^{b}$ & 2.9 & $2.0 \pm 0.5^{b}$ & & \\
\hline [5] & $\mathrm{P}$ & $\mathrm{A} 4 \mathrm{~V}$ & & & & & & & & $12 \pm 2$ & \\
\hline [6] & $\mathrm{P}$ & $\mathrm{A} 4 \mathrm{IVe}+\mathrm{sh}$ & $3.93 \pm 0.05$ & $8500^{ \pm 500}$ & $1.55_{-0.05}^{+0.06}$ & 3.9 & $2.3^{b}$ & 2.8 & $2.0^{b}$ & & \\
\hline [7] & $\mathrm{P}$ & $\mathrm{A} 7.5-8 \mathrm{Ve}$ & 3.86 & $\approx 7300$ & $1.42_{-0.07}^{+0.04}$ & 3.7 & $2.1^{c}$ & 3.3 & $5^{c}$ & & $18_{-11}^{+14}$ \\
\hline \multirow[t]{2}{*}{ [8] } & $\mathrm{P}$ & A7.5-8Ve [7] & & $\approx 7300[7]$ & 1.42 [7] & 3.7 & $2.2 \pm 0.1^{e}$ & 3.3 & $2^{e}$ & & \\
\hline & S & K3 & 3.675 & 4730 & 0.42 & 3.9 & $1.7 \pm 0.1^{e}$ & 2.5 & $2^{e}$ & & \\
\hline [9] & $\mathrm{P}$ & A7.75 [7] & 3.88 & 7648 & 1.46 & 3.8 & $2.2^{d}$ & 3.1 & $\approx 2^{d}$ & & \\
\hline [10] & $\mathrm{P}$ & & 3.90 & 8000 & & 4.5 & & & & $10 \pm 1$ & \\
\hline [11] & $\mathrm{P}$ & $\mathrm{A} 4-5 \mathrm{Ve}$ & & & & & & & & & \\
\hline [12] & $\mathrm{P}$ & & & & & & & & & & $23_{-8}^{+9}$ \\
\hline
\end{tabular}

Notes. 2nd column: $\mathrm{P}$ corresponds to the stellar parameters of the primary and $\mathrm{S}$ to those of the secondary. Values in italics (log $g$ and $R / R_{\odot}$ ): computed from universal gravitation and Stefan-Boltzmann laws. References: [1] Hu et al. (1989), [2] Hu et al. (1991), [3] Brown et al. (1997), [4] van den Ancker et al. (1997), [5] Donati et al. (1997), [6] van den Ancker et al. (1998), [7] Grady et al. (2004), [8] Böhm et al. (2004), [9] Luhman (2004), [10] Acke \& Waelkens (2004), [11] Lyo et al. (2008), [12] Böhm et al. (2006). Mass and ages are determined using the model isochrones and isomass of ${ }^{(a)}$ Iben \& Renzini (1984), ${ }^{(b)}$ Palla \& Stahler (1993), ${ }^{(c)}$ Siess et al. (2000), ${ }^{(d)}$ Palla \& Stahler (1999), ${ }^{(e)}$ Palla \& Stahler (2001).

additional lines from the secondary) nor the asymmetries or the emission components in the line profiles. Using the solar abundances by Anders \& Grevesse (1989), these authors found a solar-like global metallicity $([\mathrm{M} / \mathrm{H}]=+0.06 \pm 0.05)$ and a solar abundance for several chemical elements including $\mathrm{Fe}([\mathrm{Fe} / \mathrm{H}]=$ $+0.09 \pm 0.19$ where $\left.[\mathrm{Fe} / \mathrm{H}]=\log \left(\frac{N_{\mathrm{Fe}}}{N_{\mathrm{H}}}\right)_{\mathrm{HD} 104237}-\log \left(\frac{N_{\mathrm{Fe}}}{N_{\mathrm{H}}}\right)_{\odot}\right)$.

Donati et al. (1997) measured a $v$ sini of $12 \pm 2 \mathrm{~km} \mathrm{~s}^{-1}$, which suggests either that HD 104237 is viewed close to pole-on or that it is a moderate rotator. Böhm et al. (2006) detected a modulation of the $\mathrm{H} \alpha$ line profile with a period of $100 \pm 5 \mathrm{~h}$ and suggested a rotational origin, yielding an inclination of $23_{-8}^{\circ+9}$. This value of $P_{\text {rot }}$ was confirmed by Testa et al. (2008) in an X-ray study of the HD 104237 system.

Concerning magnetism, Donati et al. (1997) detected a weak field of about $50 \mathrm{G}$ in HD 104237, but a later survey by Wade et al. (2007) could not confirm it at this stage.

\subsection{Previous pulsational analysis}

Following the first discovery of pulsations in HD 104237 by Donati et al. (1997) and confirmation by Kurtz \& Müller (1999), Böhm et al. (2004) obtained in 1999 and 2000 an extensive highresolution $(R \approx 35000)$ spectroscopic data set of HD 104237 at the $1.9 \mathrm{~m}$ Radcliffe telescope of the SAAO (see Sect. 3). The analysis of the resulting high-quality radial velocity curve allowed them to detect for the first time by spectroscopic means multi-periodic oscillations in a PMS star (eight frequencies in the 1999 data set and five in the 2000 data set). Five frequencies of 1999 were identified with frequencies of 2000, between 28.50 and $35.60 \mathrm{~d}^{-1}$. The three last frequencies of 1999 ( $f_{6}$ to $f_{8}$ ) were considered as less certain. The frequency differences of the eight, respectively five frequencies detected by Böhm et al. (2004) were smaller than the estimated large separation ( $\Delta v_{0} \approx 40-45 \mu \mathrm{Hz}$ ), leading the authors to conclude on the nonradial nature of at least some of the pulsation modes detected in their work. More generally, no regular frequency pattern was clearly identified in the pulsational behaviour of HD 104237.
Using the results of Böhm et al. (2004), Dupret et al. (2006, 2007) found in a preliminary seismic modeling of HD 104237 that it is difficult to properly fit the observed frequencies with theoretical frequencies computed from models with $T_{\text {eff }}$ and $\log \left(L_{\star} / L_{\odot}\right)$ presented in van den Ancker et al. (1998) and Grady et al. (2004). An important result Dupret et al.'s conclusion that HD 1043237 could in fact not be a typical $\delta$ Scuti type pulsator, because the $\mathrm{p}$ modes order corresponding to the observed frequencies are too high to be excited by the standard excitation mechanism of $\delta$ Scuti type variables, namely a $\kappa$-driving in the He II partial ionization zone.

\section{SAAO spectroscopic observations and data reduction}

The analysis presented in this article is based on parts of the data set described in Böhm et al. (2004), namely the highresolution quasi-continuous spectroscopic observations obtained in April 1999 and April 2000 at the 1.9 m SAAO (South African Astronomical Observatory) Radcliff telescope; all data were obtained with the GIRAFFE fiber-fed echelle spectrograph $(R \approx$ 35000 , visible domain $430-690 \mathrm{~nm}, t_{\exp }=5 \mathrm{~min}$, signal-tonoise ratio $(\mathrm{S} / \mathrm{N})$ at $550 \mathrm{~nm}$ of $50-70 \mathrm{pix}^{-1}$ for each exposure). Data reduction was carried out following standard reduction procedures using the "ESPRIT" spectroscopic reduction package (Donati et al. 1997). The least-squares deconvolution (LSD) method as described in Donati et al. (1997) which uses the multiplex gain of more than 500 lines present in the spectrum of this star, allowed us to obtain high $\mathrm{S} / \mathrm{N}$ equivalent photospheric profiles. High-precision radial velocity correction was achieved using the positions of more than 100 narrow telluric vapor lines. All spectra were shifted to the heliocentric rest wavelength and calibrated to a final intrinsic precision estimated to be around $100 \mathrm{~m} \mathrm{~s}^{-1}$ for this data set.

Our search for direct signatures of non-radial pulsations in the equivalent photospheric profiles was carried out based on the data collected during the nights of April 22 to 26, 1999, while the fundamental parameters were determined with data taken at the periastron night of April 12, 2000, ensuring 
Table 2. Log of observations.

\begin{tabular}{lrrl}
\hline \hline Date & $\begin{array}{r}\text { Time series } \\
\text { duration (h) }\end{array}$ & $\begin{array}{r}\text { Number of } \\
\text { spectra }\end{array}$ & $\begin{array}{c}\text { S/N (pixel } \\
\text { at 550 nm }\end{array}$ \\
\hline 1999-Apr.-22 & 8.2 & 74 & $55-120$ \\
1999-Apr.-23 & 10.2 & 91 & $40-90$ \\
1999-Apr.-24 & 10.8 & 136 & $30-100$ \\
1999-Apr.-25 & 10.9 & 156 & $30-60$ \\
1999-Apr.-26 & 7.7 & 58 & $30-80$ \\
\hline 2000-Apr.-12 & 3.6 & 35 & $40-60$ \\
\hline
\end{tabular}

a maximum separation of both binary components of around $60 \mathrm{~km} \mathrm{~s}^{-1}$. This avoided pollution of the primary by the much fainter HD 104237b spectrum as far as possible. The log of the observations is summarized in Table 2.

\section{Direct detection and identification of non-radial pulsations}

The most direct way to perform mode identification in multiperiodically oscillating stars is the analysis of line-profile variations of rotationally-broadened high-resolution photospheric profiles. The maximum attainable degree $\ell$ increases with the ratio $v \sin i$ to the resolved spectral element $(\Delta v=c / R)$, the parameters of HD 104237 and the instrumental set-up indicating a detection limit of $\ell_{\max }$ on the order of 3 .

Each LSD profile was corrected for its radial velocity (orbital motion and centroid shifts mainly caused by radial pulsations) and a mean LSD profile, averaged over the whole run, was individually subtracted. The residuals show profile variations that are clearly indicative of non-radial stellar pulsations, with differential amplitudes on the order of $1.5 \%$ of the continuum. Also, the dynamical spectra of Fig. 1 show that beating between nearby frequencies occurs, which leads to the conclusion that there is probably more than one non radial pulsation (NRP; the night of April 26 is not represented because of shorter coverage). Figure 2 shows the differential amplitude variations at line center, extracted by taking the median value of the three central velocity bins of each spectrum, one bin corresponding to $4 \mathrm{~km} \mathrm{~s}^{-1}$.

\subsection{Central profile analysis with SigSpec and Period04}

We carried out a frequency analysis of the time-series using the Period04 package (Lenz \& Breger 2005) in parallel with the Sigspec package (Reegen 2007). They both use, in iterative process, combinations of discrete Fourier transform (DFT) and least-squares fitting algorithms to extract the frequencies, amplitudes and phases of multi-periodic signals in not-equally spaced data sets. To avoid at maximum low-frequency night-to-night variations, we corrected the time series by subtracting a nightly average before performing the analysis. Still, by doing so, some low frequencies persisted. The results of this analysis are shown in Table 3. As described in Böhm et al. (2004), the uncertainty in the extracted frequency value can be estimated in different ways. The most conservative approach is measuring of the FWHM of the main lobe of the spectral window function (Ripepi et al. 2003), corresponding here to $\Delta f=0.31 \mathrm{~d}^{-1}$. Figure 3 shows the amplitude power spectrum including all strong peaks around 29-36 d $\mathrm{d}^{-1}$, the noise levels were determined after prewithening of the nine first frequencies. Figure 4 shows that even $F_{9}$ has a significance level of above $99.9 \%$, following the approach described in Breger et al. (1993) and Kuschnig et al. (1997). We arbitrarily decided to stop the iterative procedure for amplitude values below $0.1 \%$. The verification of the results with SigSpec revealed exactly the same frequencies and amplitudes, all detected frequencies $F_{1}$ to $F_{10}$ having a significance level between 66.5 and 6.3, respectively. No combined frequency is present in the power spectrum, following the analysis with the Combine program by Reegen (2007). We did not consider the frequencies below approximately $5 \mathrm{~d}^{-1}$, because differential calibration problems from one night to the next might persist, i.e. this excludes $F_{4}, F_{7}, F_{8}$, and $F_{10}$. By applying the error estimate by Ripepi et al. (2003), all remaining frequencies (" $F$ ") could be identified with the frequencies (" $f$ ") determined by radial velocity studies on the same data set in Böhm et al. (2004). $F_{3}$ is slightly outside the error bar, and the $1.0 \mathrm{~d}^{-1}$ difference between $F_{9}$ and $F_{3}$ indicates that we most likely deal with an alias of the same frequency. The order of the first two dominant frequencies is inverted depending on if radial velocity or central depth variations are analyzed, probably because of the prior radial velocity recentering.

\subsection{Mode identification with the Fourier 2D method}

A direct method of analyzing the non-radial pulsation modes present in the LSD-spectra time series is applying the Fourier 2D method on line-profile variations (Kennelly et al. 1993; Kennelly 1994; Kennelly et al. 1996). This technique analyzes the complex pattern present in the line profiles by computing a 2D Fourier transform in time and Doppler space. To do this, we performed an interpolation of each profile on a grid representing stellar longitudes, transforming velocities across the line profile into longitudes on the stellar equator using the relation $\Delta v=v \sin i \sin \phi . \Delta v$ is here the velocity position within the LSD profile with respect to the rest wavelength of the star and $\phi$ is the stellar longitude angle of the star in spherical coordinates. In the resulting $2 \mathrm{D}$ Fourier spectrum, the temporal frequencies are related here mainly to the frequencies of oscillation, while the apparent azimuthal order $\hat{\mathrm{m}}$ is related to the structure of the modes present at the stellar surface, which is not identical to the usual azimuthal order m. The original work by Kennelly (1994) showed that apparent $|\hat{m}|$ scales as $\ell+2$ for values close to zero, as $\ell+1$ for values lower than 10 and as $\ell$ for values above $10(\ell$ being the degree of the mode). We performed this F2D computation on each night of April 22 to 25 1999. A weighted combination of the nightly F2D spectra can be seen in Fig. 5 (see Böhm et al. 2009, for details of the method) and our results confirm the presence of low-degree non-radial pulsations: a peak at the dominant frequency $F_{1}$ is clearly seen with an apparent $\hat{m}$ of between 2 and 4 indicating a degree $\ell$ of between 0 and 2. Moreover, as can be seen in Table 2.3 of Kennelly (1994) (or in simulations with available online-NRP simulators), the symmetric pattern of the dynamical residual profile as seen in Fig. 1 indicates an azimuthal order $m= \pm 1$ for $F_{1}$, implying a most likely $\ell$ value of 1 or 2.

The frequency peak in Fig. 5 between 3 and $5 \mathrm{~d}^{-1}$ is again not taken into account, because nightly calibration shifts can easily introduce such variations.

A complementary analysis on the whole data set using the FAMIAS code (Zima et al. 2006; Zima 2008) is planned, but the highly asymmetric mean photospheric profile seen in HD 104237 adds complexity to the data analysis and cannot be taken into account in the present state of the code. 
A. Fumel and T. Böhm: Spectroscopic monitoring of the Herbig Ae star HD 104237. II.
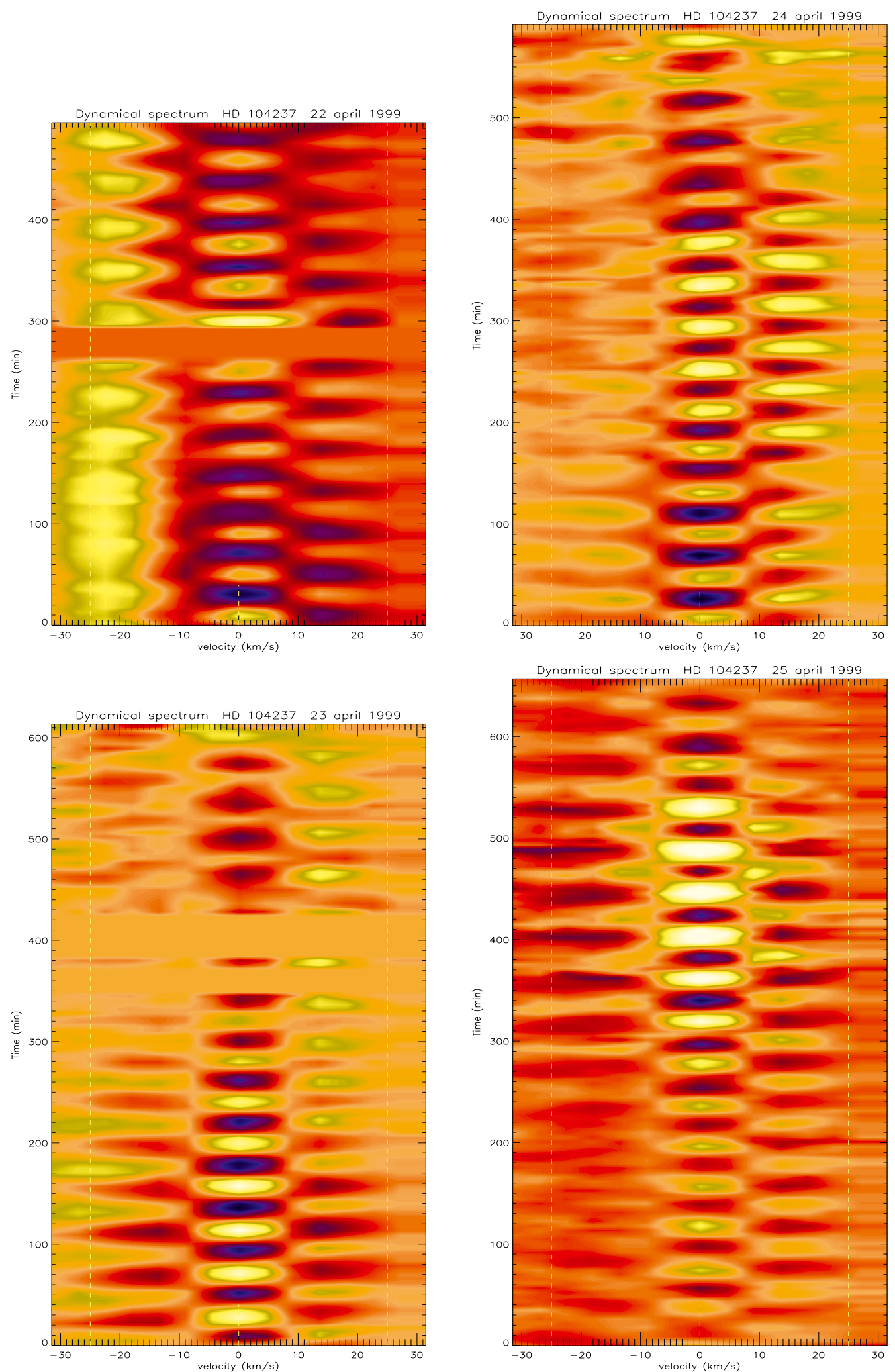

Fig. 1. Line profile variations due to the non-radial pulsations of HD 104237. The deviations from the mean intensity are displayed for four different nights (top left: April 22, 1999, bottom left: April 23, 1999, top right: April 24, 1999, bottom right: April 25, 1999). Dashed lines represent the limits of the equivalent profile, an additional broadening agent generates wings reaching almost $\pm 2 v \sin i$, i.e. twice the width of a pure rotational profile at continuum level.

\section{Optimized spectral continuum determination}

Our next step, the fundamental parameter redetermination, required summing up of individual spectra obtained during a periastron night, the goal being a resulting spectrum with very high $\mathrm{S} / \mathrm{N}$. Each individual spectrum had to undergo an optimized continuum normalization as an essential prerequisite. An automatic code was developed, because the case of HD 104237 


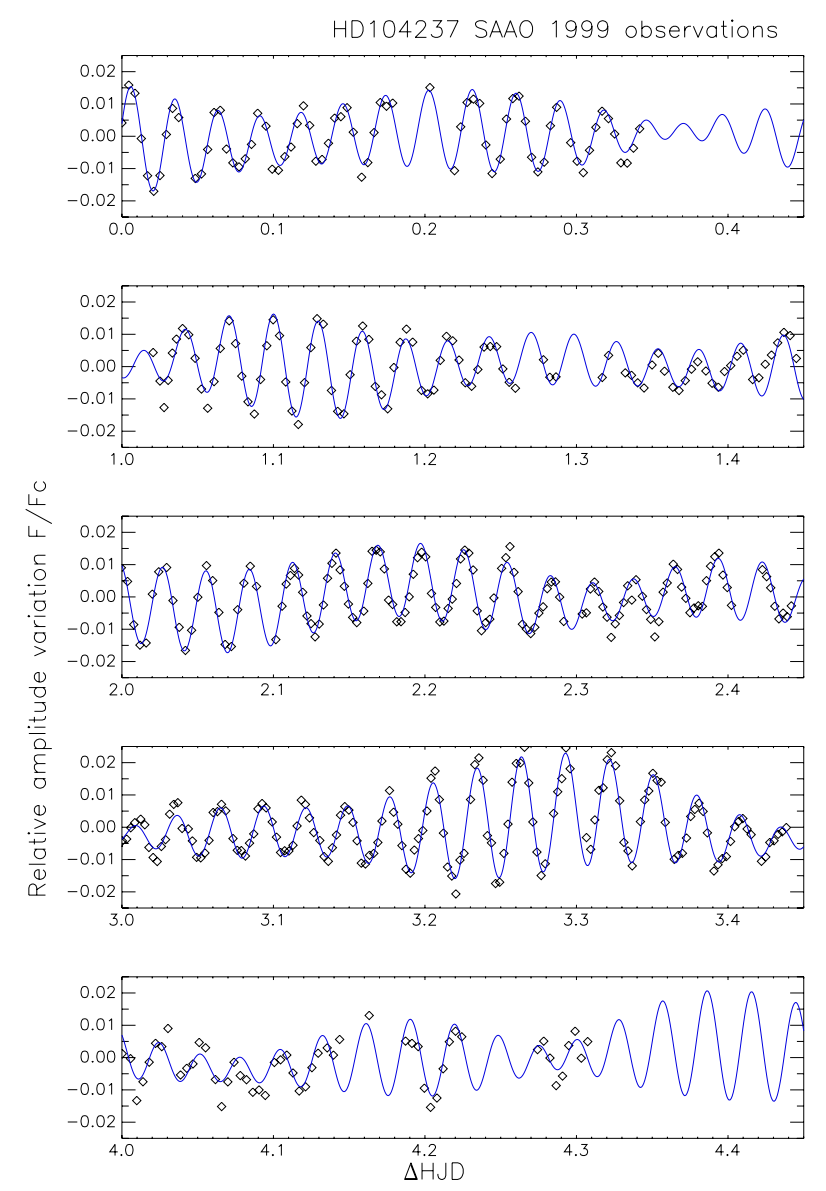

Fig. 2. Amplitude variation of the central velocity bins of the residual LSD profile on the nights Apr. 22 to 26, 1999. Superimposed is a fit of the data corresponding to the result of the frequency analysis as presented in Table 3.

Table 3. Frequencies determined with Period04.

\begin{tabular}{lrrcc}
\hline \hline$\#$ & $\begin{array}{r}F_{\mathrm{P} 04} \\
\left(\mathrm{~d}^{-1}\right)\end{array}$ & $\begin{array}{r}F_{\mathrm{P} 04} \\
(\mu \mathrm{Hz})\end{array}$ & $\begin{array}{c}A_{\mathrm{P} 04} \\
\left(F / F_{\mathrm{c}}\right)\end{array}$ & $f_{\mathrm{B} 04}(|\delta \mathrm{f}|)$ \\
\hline$F_{1}$ & 35.60 & 412.04 & 0.0089 & $f_{2}(0.01)$ \\
$F_{2}$ & 33.74 & 390.51 & 0.0033 & $f_{1}(0.12)$ \\
$F_{3}$ & 32.25 & 373.26 & 0.0030 & $f_{6}(0.36)$ \\
$F_{4}$ & 4.47 & 51.74 & 0.0024 & \\
$F_{5}$ & 31.08 & 359.72 & 0.0028 & $f_{4}(0.12)$ \\
$F_{6}$ & 34.00 & 393.52 & 0.0019 & $f_{5}(0.14)$ \\
$F_{7}$ & 1.94 & 22.45 & 0.0018 & \\
$F_{8}$ & 2.72 & 31.48 & 0.0014 & \\
$F_{9}$ & 29.48 & 341.20 & 0.0011 & $f_{3}(1.0)$ \\
$F_{10}$ & 5.17 & 59.84 & 0.0010 & \\
\hline
\end{tabular}

Notes. The different columns are: (1) number, (2) frequency in $\mathrm{d}^{-1}$ and (3) in $\mu \mathrm{Hz}$, (4) Amplitude in $\left(F / F_{\mathrm{c}}\right)$ (flux variation with respect to the average profile), (5) identification with frequencies as of Böhm et al. (2004), and absolute shift in $\mathrm{d}^{-1}$.

proved to be quite complicated owing to the pollution by the spectrum of its secondary companion, but also because of emission components and variability observed in numerous absorption lines. As a first step, we determined the effective continuum location within each composite spectrum. To do so, absorption line lists were extracted from the Vienna Atomic Line Database (VALD; Piskunov et al. 1995; Ryabchikova et al. 1997; Kupka et al. 1999, 2000) corresponding to the approximate fundamental parameters of each component. For each individual spectrum,

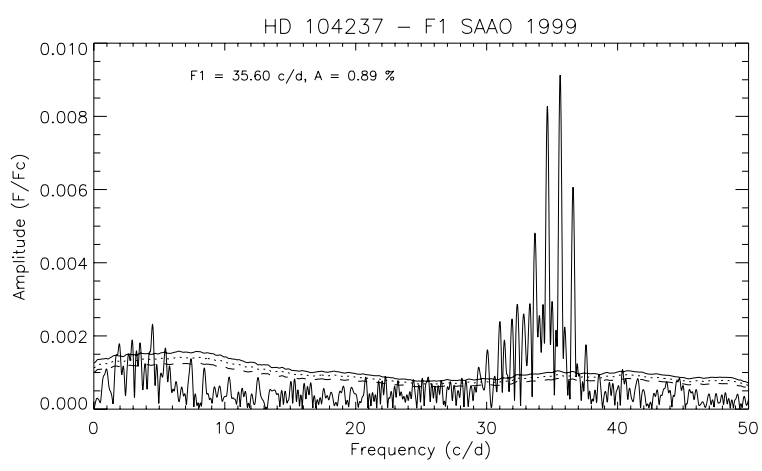

Fig. 3. Periodogram of the line center variation as shown in Fig. 2, without prewithening. The large bulk of frequencies around $29-36 \mathrm{~d}^{-1} \mathrm{can}$ be seen, as well as the 3.2,3.6 and 4.0 mean-ampitude level of the noise determined by prewithening $F_{1}$ to $F_{9}$, corresponding to a $90 \%, 99 \%$ and 99.9\% significance level, respectively.

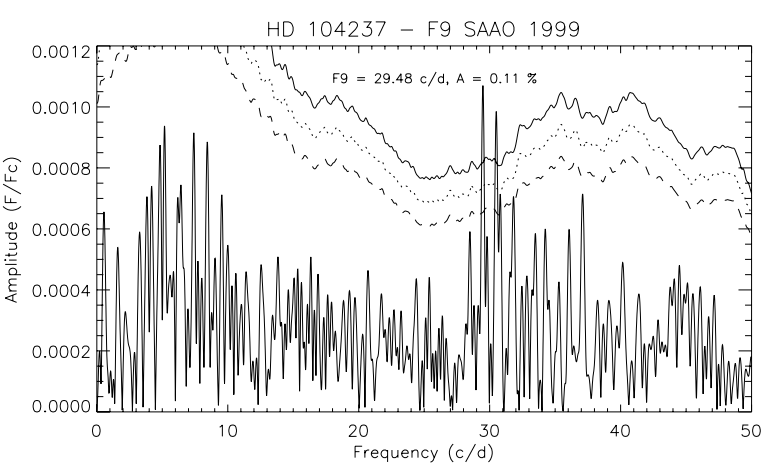

Fig. 4. Same figure as Fig. 3, but prewithened by $F_{1}$ to $F_{8}$. As shown, $F_{9}$ is present, well above the $99.9 \%$ confidence level.

these grids were shifted by the precise orbital velocities at the acquisition time. A wavelength-dependant luminosity-ratio (black-body approximation) of both components was calculated and a depth criterion applied. The rotational broadening was taken into account. Finally, the elimination of unsuited, "active" spectral line regions let us determine true continuum regions. The division by a fourth degree polynomial fitted through the remaining continuum areas of each order normalized them and was followed by their weighted concatenation, taking into account the local $\mathrm{S} / \mathrm{N}$ in the overlapping regions between adjacent orders (excluding the broad $\mathrm{H} \alpha$ and $\mathrm{H} \beta$ line areas, which remained unnormalized). In a last step, all obtained individually normalized spectra of the night were added, weighted by their corresponding average $\mathrm{S} / \mathrm{N}$, after recentering them on their individual primary component rest velocity.

The resulting gain in $\mathrm{S} / \mathrm{N}$ of our reference "periastron" spectrum (see Fig. 6) was of about a factor of 6 , the $S / N$ reaching almost 400 per resolved element at $550 \mathrm{~nm}$.

\section{Fundamental parameter determination}

\subsection{Method}

Since $T_{\text {eff }}, \log g$, chemical abundances $\left(\log A_{\mathrm{E}}=\log \left(\frac{N_{\mathrm{E}}}{N_{\mathrm{H}}}\right)\right.$ for an element $\mathrm{E}$ ), and microturbulent velocity $v_{\text {micro }}$ are interdependant and simultaneously affect the depth, width, and shape of spectral absorption lines, they have to be determined simultaneously. Numerous methods exist for this analysis, but very few of them are suitable in the particular case of HD 104237. Owing to the non-standard shape of HAeBe spectral energy distributions 
A. Fumel and T. Böhm: Spectroscopic monitoring of the Herbig Ae star HD 104237. II.

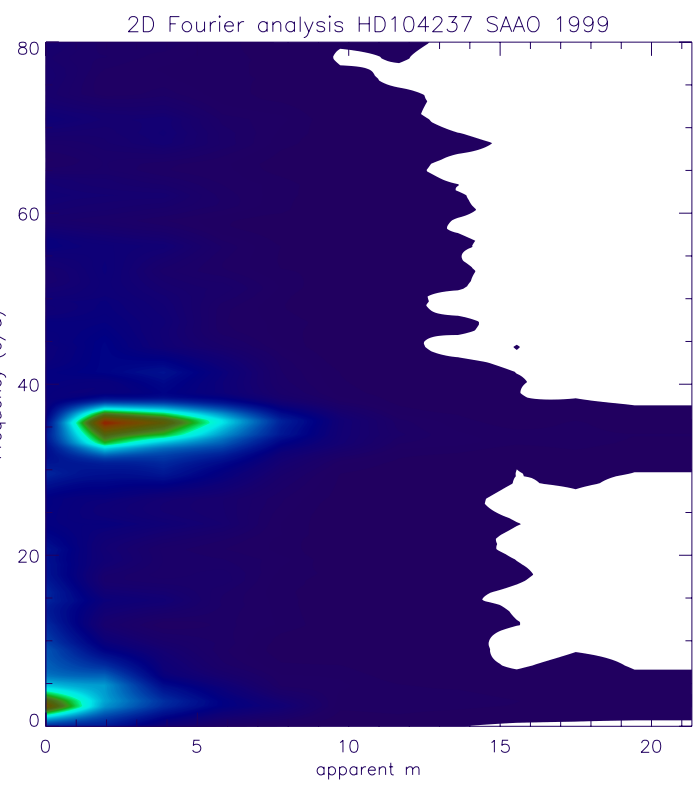

Fig. 5. Combined F2D analysis of HD 104237 for the nights of April 22 to 25,1999 .

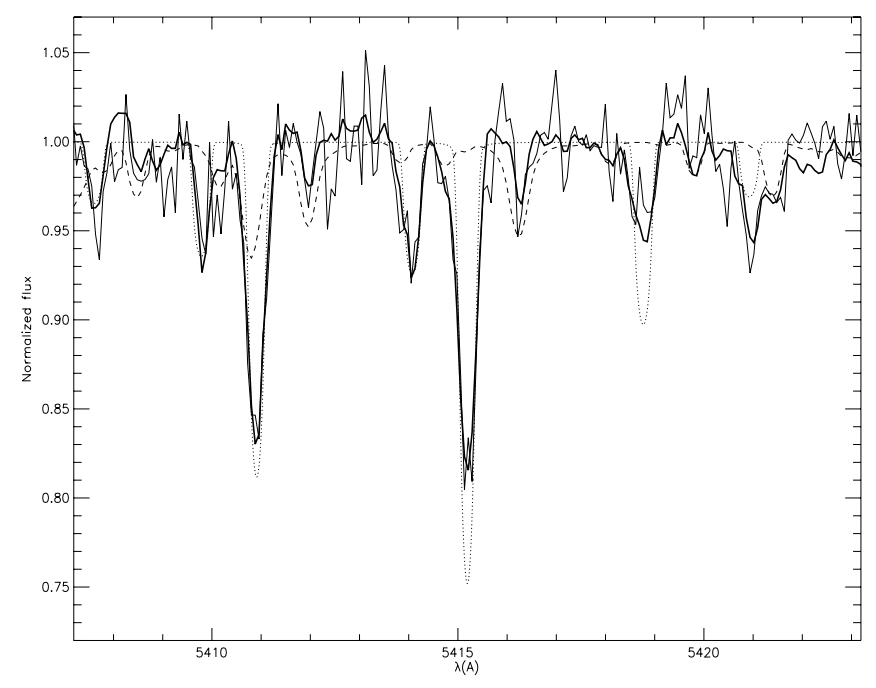

Fig. 6. Individual (thin line) and summed (thick line) normalized spectrum of the periastron night around one of the selected lines (Fe I $5415.1920 \AA$ ). Synthetic spectra with $T_{\text {eff }}=8500 \mathrm{~K}$ (dotted line) and $T_{\text {eff }}=4750 \mathrm{~K}$ (dashed line) were overplotted $\left(\log g=4.0, v_{\text {micro }}=\right.$ $2 \mathrm{~km} \mathrm{~s}^{-1}$ solar abundances for both spectra), showing the location of the individual lines. The secondary's spectrum was corrected by the local luminosity ratio. Neither fundamental parameters nor local luminosity ratios are perfectly tuned at this stage.

(IR excesses, UV depletion), classical photometric determination of $T_{\text {eff }}$ have to be considered with caution. Another frequently used method, the stellar parameter determination using Balmer lines had to be excluded because of core emission in the line and/or P Cygni profiles, but also on a technical side because of normalization problems for high-dispersion echelle spectra. Techniques based on line ratios for determining $T_{\text {eff }}$ (see e.g. Sousa et al. 2010) could not be applied owing to the too few workable lines (see Sect. 6.1.3). Photospheric lines of HD 104237 reveal additional broadening agents that are difficult to model, which led us to discard direct spectrum fitting.
Our finally adopted method consists of comparing the EW of selected lines present in our reference spectrum with those extracted from a fine 3D-grid of synthetic spectra (the dimensions being $T_{\text {eff }}, \log g$ and iron abundance). The high activity level of this HAe star (emission, variability, line asymmetry) and its binarity make a reliable determination of fundamental parameters in this case very challenging.

\subsubsection{Correction of the secondary's spectral contribution}

Since our main goal was to determine the fundamental parameters of the primary component, we had to correct for the polluting spectroscopic contribution of the secondary component, HD 104237b. Indeed, although the secondary is more than $1 \mathrm{dex}$ fainter than the primary component, it is also approximately $4000 \mathrm{~K}$ cooler and contributes with strong metallic absorption lines, leading to systematic contamination of primary lines and even lowering the continuum level in some parts of the spectrum. To free the observed spectrum from this effect, a synthetic spectrum corresponding to the secondary component's contribution was substracted from the observed spectrum. A $\chi^{2}$ minimization indicated a likely secondary $T_{\text {eff }}$ value of $4500 \mathrm{~K}$. Still, residual systematic uncertainties of the monochromatic luminosity ratio and the intrinsic stellar parameter selection of the secondary do certainly persist and are difficult to take into account.

\subsubsection{Construction of a 3D-grid of synthetic spectra}

The fundamental parameter determination method we adopted required the construction of a 3 -dimensional grid $\left(T_{\mathrm{eff}}, \log g\right.$, $\log A_{\mathrm{Fe}}$ ) of synthetic spectra based on line catalogs provided by the VALD database as input of the SYNTH3 spectrum synthesis code (Kochukhov 2007), each spectrum covering a wavelength range from $4400 \AA$ to $7000 \AA$. The SYNTH3 code assumes local thermodynamic equilibrium (LTE) and a plane-parallel hydrostatic stellar model atmosphere. For early-type stars input models are provided by the well-known Kurucz program suite. We selected solar abundance atmosphere models from the grid after checking that the effect of an input model that contains metallicities slightly different from solar is of secondary importance on the calculated synthetic spectrum.

The grid boundaries covered previously determined parameters: $7500 \mathrm{~K} \leq T_{\text {eff }} \leq 9500 \mathrm{~K}$ and $3.5 \leq \log g \leq 4.5$. Since it is not uncommon to find HAeBe stars that simultaneously show some elements in suprasolar abundance in their spectra, whereas other ones are subsolar (see e.g. Acke \& Waelkens 2004) without a particular trend in terms of global metallicity, we decided to modify only the $\mathrm{Fe}$ abundance in our VALD requests. $\log A_{\mathrm{Fe}}$ values comprised between -4.84 and -4.00 seemed to be reasonable, i.e., of $[\mathrm{Fe} / \mathrm{H}]$ values between -0.3 and +0.54 with respect to the solar Fe abundance given in VALD $\left(\log A_{\mathrm{Fe}_{\odot}}=\right.$ -4.54). Typical values of $v_{\text {micro }}$ found in the literature were 2 $3 \mathrm{~km} \mathrm{~s}^{-1}$ (see e.g. Acke \& Waelkens 2004; Guimarães et al. 2006; Catala et al. 2007). We therefore fixed a value of $2 \mathrm{~km} \mathrm{~s}^{-1}$ for the microturbulent velocity HD 104237. The resulting grid was refined by interpolating between the models that were initially spaced by steps of $250 \mathrm{~K}$ and $0.5(\log g)$.

\subsubsection{Photospheric line selection and EW measurements}

The photospheric absorption lines retained for our study had to satisfy several selection criteria. Only lines were kept when 
Table 4. Characteristics of absorption lines selected for the fundamental parameter determination.

\begin{tabular}{lccccccr}
\hline \hline$\lambda_{\mathrm{c}}(\AA)$ & el.+ion. & $\chi_{\mathrm{eV}}$ & $\log g f$ & $\overline{S / N}$ & $\mathrm{EW}_{\text {obs }}(\mathrm{m} \AA)$ & $\sigma_{\text {obs }}(\mathrm{m} \AA)$ & $\sigma_{\text {rel }}(\%)$ \\
\hline 4485.675 & Fe I & 3.686 & -1.020 & 217 & 16.1 & 2.6 & 15.9 \\
4602.941 & Fe I & 1.485 & -2.209 & 245 & 39.3 & 4.0 & 10.1 \\
5049.819 & Fe I & 2.279 & -1.355 & 268 & 51.9 & 3.9 & 7.6 \\
5132.669 & Fe II & 2.807 & -4.094 & 275 & 28.0 & 4.0 & 14.5 \\
5393.167 & Fe I & 3.241 & -0.715 & 289 & 46.7 & 3.1 & 6.6 \\
5415.192 & Fe I & 4.386 & 0.642 & 285 & 97.9 & 4.4 & 4.5 \\
5434.523 & Fe I & 1.011 & -2.122 & 273 & 51.9 & 3.3 & 6.3 \\
5576.089 & Fe I & 3.430 & -1.000 & 272 & 35.2 & 3.5 & 9.8 \\
6084.111 & Fe II & 3.199 & -3.881 & 245 & 18.2 & 4.0 & 21.8 \\
6400.000 & Fe I & 3.602 & -0.290 & 238 & 51.3 & 4.7 & 9.2 \\
\hline
\end{tabular}

Notes. Column are: (1) central wavelengh, (2) ion, (3) excitation potential (eV), (4) log of statistical weight $\times$ oscillator strength of the line transition, (5) local mean $\mathrm{S} / \mathrm{N}$ ratio, (6) observed equivalent width, (7) absolute and (8) relative uncertainty on the equivalent width.

they existed in all line lists of our grid above a minimum threshold. We excluded blends with other than iron lines, and avoided pollution by potential residuals of the secondary spectrum. Variable and emission lines were rejected. Lines had to be strong enough with respect to local noise to minimize relative uncertainties on EW and to be able to neglect residual contribution from very faint lines of the primary's and the secondary's spectra, inherent to the extraction threshold of VALD requests. As a result, we kept weak and moderatly strong lines $\left(15 \mathrm{~m} \AA \leq E W_{\text {obs }} \leq 100 \mathrm{~m} \AA\right)$. Moreover, the nearby continuum of the line had to be free of emission components. We had to verify that selected lines cover a wide variety of excitation potentials, ionization stages, and wavelengths. Atomic parameters were verified, in particular log $g f$ values (comparison with Fuhr \& Wiese 2006; Meléndez \& Barbuy 2009). This rigorous selection resulted in a set of only ten spectral lines (eight Fe I lines and two Fe II lines) which are listed in Table 4.

Equivalent width measurements of our selected lines were carried out using a trapezoidal integration. For consistency, the identical extraction limits were used for the integration of a given line from the observed spectrum and the synthetic spectra. To extract errors on EW values, we assumed that they mainly came from local averaged photon noise (multiplied by the line integration width).

\section{2. $T_{\text {eff }}, \log g$, and Fe abundance determination}

We then computed the degree of similarity between our observed spectrum and all synthetic spectra of our 3D-grid by means of the ten previously measured EW. A maximum-likelihoodestimation was performed by minimizing a reduced merit function $S_{\text {red }}$ following Press et al. (1992).

A preliminary computation of the reduced merit function whithin the whole 3D-grid of synthetic spectra, whithout any additional constraint, yielded a $T_{\text {eff }}$ value of $8775 \mathrm{~K}$, a $\log g$ value of 4.2 , and a $\log A_{\mathrm{Fe}}$ value of -4.25 , i.e. $[\mathrm{Fe} / \mathrm{H}]=+0.29$. With a confidence level of $68.3 \%$ we found the following uncertainties: $525 \mathrm{~K}$ on $T_{\text {eff }}, 0.7$ on $\log g$ and 0.35 on $\log A_{\mathrm{Fe}}$. With a confidence level of $95.4 \%$ we determined the uncertainties to be $700 \mathrm{~K}$ on $T_{\text {eff }}$ and 0.48 on $\log A_{\mathrm{Fe}}$, the low sensitivity to $\log g$ does not allow the determination of the $95.4 \%$ confidence level for $\log g$ within our parameter space.

The lack of selected lines is responsible for the quite large uncertainties in the 3D-parameter space. Therefore we added the additional physical constraint provided by the excitation

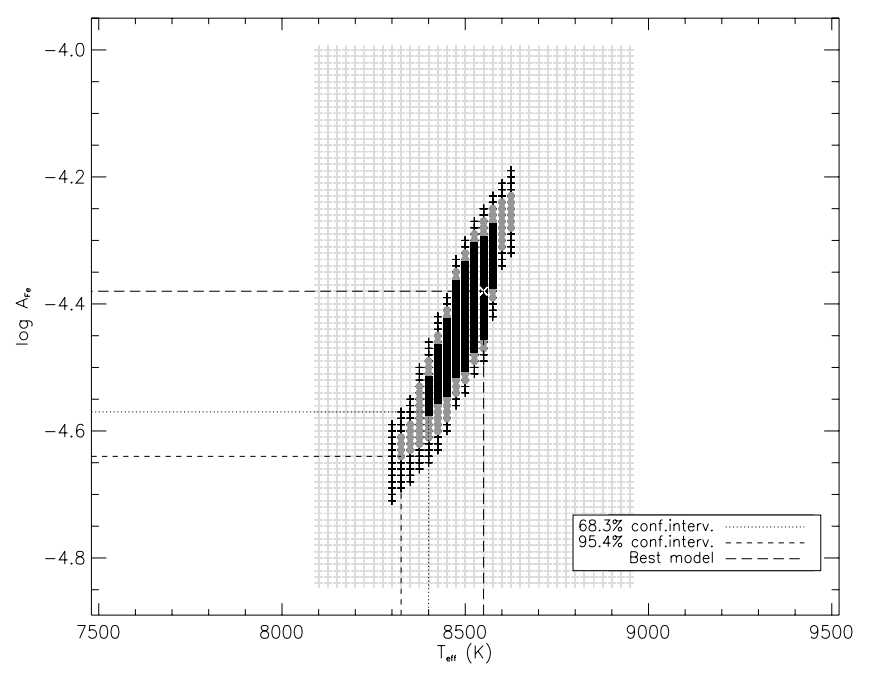

Fig. 7. 2D-repartition of the $S_{\text {red }}$ values as a function of $T_{\text {eff }}$ and $\log A_{\mathrm{Fe}}$. The white $\mathrm{X}$ represents the best model $\left(T_{\text {eff }}=8550 \mathrm{~K}, \log g=\right.$ $\left.3.9, \log A_{\mathrm{Fe}}=-4.38\right)$. Boundaries are shown for the $68.3 \%$ and $95.4 \%$ confidence intervals. Gray crosses show the parameter space investigated, after selecting models that satisfied the excitation potential criterium.

Table 5. Fundamental parameters of HD 104237 determined in this study.

\begin{tabular}{lccc}
\hline \hline $\begin{array}{l}\text { Stellar } \\
\text { param. }\end{array}$ & $\begin{array}{c}\text { Best model } \\
\text { param. values }\end{array}$ & $\begin{array}{c}68.3 \% \\
\text { conf. int. }\end{array}$ & $\begin{array}{c}95.4 \% \\
\text { conf. int. }\end{array}$ \\
\hline$T_{\text {eff }}(\mathrm{K})$ & 8550 & \pm 150 & \pm 225 \\
$\log g$ & 3.9 & \pm 0.3 & \pm 0.4 \\
$\log A_{\mathrm{Fe}}$ & -4.38 & \pm 0.19 & \pm 0.26 \\
\hline
\end{tabular}

potential: if $T_{\text {eff }}$ is well determined, all spectral lines corresponding to a specified ion, and with a given excitation potential, should provide the same abundance determination. We therefore only kept stellar models yielding a zero $\pm 1 \sigma$ slope regression line (through the distribution $\log A_{\mathrm{Fe}}=f(\chi)$, with $\chi$ being the excitation potential). Recalculating a minimum value of the reduced merit function $S_{\text {red }}$ for this subset of models yielded significantly more precise parameters: $T_{\text {eff }}=8550 \mathrm{~K}, \log g=3.9$ and $\log A_{\mathrm{Fe}}=-4.38([\mathrm{Fe} / \mathrm{H}]=+0.16)$. Surface gravity $\log g$ is the less accurately determined stellar parameter because of the lower sensibility of the selected lines to this parameter. The results are shown in Fig. 7 and summarized in Table 5. 


\section{Discussion and conclusions}

We presented an in-depth analysis of the prototype Herbig Ae star HD 104237. The star was previously identified as a PMS $\delta$ Scuti pulsator, and the comprehensive line profile analysis of the extended quasi-continuous high-resolution spectroscopic time series obtained at SAAO from April 22-26, 1999 has confirmed the main frequencies that were discovered by radial velocity analysis in Böhm et al. (2004). For the first time, the less than $1.5 \%$ continuum level variations of the equivalent photospheric LSD profiles were studied and a direct confirmation of the presence of at least one non radial pulsations could be performed. Based on a Fourier 2D analysis we identified the dominant mode as an azimuthal order $m= \pm 1$, and a most likely $\ell$ value of 1 or 2 . Since HD 104237 is a moderate rotator with $v \sin i$ of only $12 \pm 2 \mathrm{~km} \mathrm{~s}^{-1}$ it will be difficult to access to additional mode identifications in the future. A potential approach would be to organize multi-site continuous observations with significantly higher resolution echelle spectrographs, ideally with $R \geq 70000$. On the mode identification aspect a modification of the FAMIAS code working with asymmetric photospheric profiles would be a great progress.

To prepare a forthcoming asteroseismic modeling of this particular star we needed to redetermine very precisely its fundamental stellar parameters. Because HD 104237 is a multiple system with a nearby spectroscopic binary companion, we decided to concentrate on the periastron night 12 of April 2000, present in our data set of spectra acquired at SAAO (and described in Böhm et al. 2004). To combine all spectra of this particular night into one high $\mathrm{S} / \mathrm{N}$ reference spectrum, we developed an optimized spectral continuum determination tool. As a result, the reference spectrum had an $\mathrm{S} / \mathrm{N}$ value of close to 400 per resolved element at $550 \mathrm{~nm}$.

The detailed study of the fundamental stellar parameters has provided values of $T_{\mathrm{eff}}=8550 \pm 150 \mathrm{~K}, \log g=3.9 \pm 0.3$ and $\log A_{\mathrm{Fe}}=4.38 \pm 0.19$ (i.e. $[\mathrm{Fe} / \mathrm{H}]=+0.16 \pm 0.19$ ), the error bars corresponding to the $68.3 \%$ confidence interval. A particular effort was made for the statistically correct determination of the associated error bars. In a review of $T_{\text {eff }}$ and $\log g$ determination methods, Smalley (2005) mentioned optimal error bars to be of $\pm 100 \mathrm{~K}$ for $T_{\text {eff }}, \pm 0.2$ for $\log g$ and on the order of 0.05 to 0.1 in abundance. Owing the high level of activity of HAeBe stars, and in the case our target star the spectroscopic binarity, it is very difficult to obtain a sufficiently large number of unpolluted, exploitable photospheric spectral lines of a given ion; the achievable uncertainties remain therefore fairly large. In addition, a wrong estimate of the contribution of the secondary, both in spectral class and in luminosity, might lead to systematic errors that are very difficult to take into account. However, we estimated these errors to be of secondary importance in our study.

Because the surface gravity was difficult to constrain in our spectroscopic approach, the associated luminosity ratio (based on the empirical mass-luminosity relation by Malkov 2007) was determined to be $\log \left(L_{\star} / L_{\odot}\right)=1.59 \pm 0.44$, a value centered on the result by van den Ancker et al. (1998), whose authors determined $\log \left(L_{\star} / L_{\odot}\right)=1.55_{-0.05}^{+0.06}$. In constrast, our spectroscopic effective temperature determination is significantly improved with $T_{\text {eff }}=8550 \pm 150 \mathrm{~K}$, again centered on the central value of van den Ancker et al. (1998), whose result was $T_{\text {eff }}=8500 \pm 500 \mathrm{~K}$, the latter error bars having been estimated. Our independent spectroscopic approach therefore tends to confirm the previously photometrically determined values by van den Ancker et al. (1998). Combining the two results

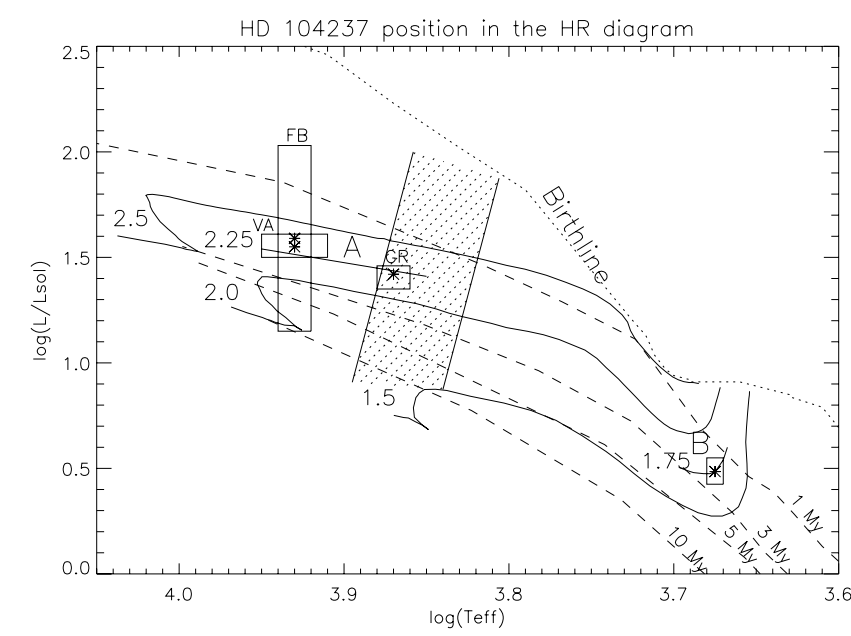

Fig. 8. New fundamental stellar parameters of the primary (A) component of HD 104237, located in the HR diagram of Böhm et al. (2004). The location of the secondary component HD $104237 \mathrm{~b}$ (B) is also indicated. The luminosity and effective temperature pairs reported in van den Ancker et al. (1998) (VA) and Grady et al. (2004) (GR) are shown in the diagram, including their estimated error bars, along with our new parameters (FB), associated with error with an $68.3 \%$ confidence interval. Evolutionary tracks $\left(1.5,2.0\right.$ and $\left.2.5 M_{\odot}\right)$ and isochrones are by Palla \& Stahler (2001). The Marconi \& Palla (1998) instability strip is represented by the shaded area.

would yield the following most likely fundamental parameters: $\log \left(L_{\star} / L_{\odot}\right)=1.55_{-0.05}^{+0.06}$, corresponding to $\log g=3.93 \pm 0.09$, and $T_{\text {eff }}=8550 \pm 150 \mathrm{~K}$. Fig. 8 shows well the intersection between our new results and previously determined parameters from van den Ancker et al. (1998). At this stage it is important to mention that error bars by van den Ancker et al. (1998) and Grady et al. (2004) might be largely underestimated.

Acke \& Waelkens (2004) determined an iron abundance of $[\mathrm{Fe} / \mathrm{H}]=+0.09 \pm 0.19$. Our new result of the abundance, $[\mathrm{Fe} / \mathrm{H}]=+0.16 \pm 0.19$, confirms the conclusion that HD 104237 might be slightly overabundant. Vick et al. (2011) recently calculated self-consistent stellar evolution models in this mass range including atomic diffusion; they concluded that for mass loss ranges above $10^{-12} M_{\odot} \mathrm{yr}^{-1}$ no surface abundance anomalies are expected. HD 104237 shows a very strong $\mathrm{H}_{\alpha}$ line with a complex profile, which tends to indicate significantly higher mass loss rates, similar to other Herbig Ae stars showing typical mass loss rates on the order of $10^{-7}-10^{-8} M_{\odot} \mathrm{yr}^{-1}$ (see e.g. Bouret $\&$ Catala 1998). Taking these new simulations into account, we conclude on a close to solar iron abundance for HD 104237, which is clearly within the error bars of both studies.

We mention at this stage that several assumptions have been made in this study, on the modeling side (LTE, plane-parrallel spectrum synthesis), on the contribution of the faint secondary (ratio of black-body fluxes), on the threshold of line selection, interpolation of models in our grid, but also the assumed photospheric line formation region of our lines of interest, among others. Although these residual errors should be negligible for our study, further improvements on line selection and analysis, such as a combined treatment of blended lines, will lead to increasingly better constrained fundamental parameters in the future.

A forthcoming article (Fumel et al., in prep.) will describe the asteroseismic modeling of the primary component of HD 104237.

Acknowledgements. The authors want to thanck N. Grevesse for interesting and fruitful discussions about stellar parameter determination and his critical advice 
about atomic parameters. Warm thanks also to $\mathrm{H}$. Carfantan for precious discussions about the statistically meaningful use of the $\chi^{2}$ quantity and his advice concerning error bar determination in multiple dimension parameter spaces. Many thanks also to F. Lignières, P. Petit and P. Fouqué for their important comments on the approach we adopted. We acknowledge the Vienna Atomic Line Data base we used for our research work. Our thoughts are going to P. Reegen for his important contribution to time series analysis.

\section{References}

Acke, B., \& Waelkens, C. 2004, A\&A, 427, 1009

Anders, E., \& Grevesse, N. 1989, Geochim. Cosmochim. Acta, 53, 197

Baines, D., Oudmaijer, R. D., Porter, J. M., \& Pozzo, M. 2006, MNRAS, 367, 737

Bertout, C., Basri, G., \& Bouvier, J. 1988, ApJ, 330, 350

Böhm, T., \& Catala, C. 1995, A\&A, 301, 155

Böhm, T., Catala, C., Donati, J.-F., et al. 1996, A\&AS, 120, 431

Böhm, T., Catala, C., Balona, L., \& Carter, B. 2004, A\&A, 427, 907

Böhm, T., Dupret., M. A., \& Aynedjian, H. 2006, Mem. Soc. Astron. It., 77, 362

Böhm, T., Zima, W., Catala, C., et al. 2009, A\&A, 497, 183

Bouret, J.-C., \& Catala, C. 1998, A\&A, 340, 163

Breger, M. 1972, ApJ, 171, 539

Breger, M., Stich, J., Garrido, R., et al. 1993, A\&A, 271, 482

Brown, A., Djie, H. R. E. T. A., Blondel, P. F. C., et al. 1997, in Accretion Phenomena and Related Outflows, ed. D. T. Wickramasinghe, G. V. Bicknell, $\&$ L. Ferrario, IAU Colloq. 163, ASP Conf. Ser., 121, 448

Catala, C. 1988, A\&A, 193, 222

Catala, C. 2003, Ap\&SS, 284, 53

Catala, C., \& Kunasz, P. B. 1987, A\&A, 174, 158

Catala, C., \& Talavera, A. 1984, A\&A, 140, 421

Catala, C., Czarny, J., Felenbok, P., \& Praderie, F. 1986a, A\&A, 154, 103

Catala, C., Felenbok, P., Czarny, J., Talavera, A., \& Boesgaard, A. M. 1986b, ApJ, 308, 791

Catala, C., Alecian, E., Donati, J.-F., et al. 2007, A\&A, 462, 293

Donati, J., Semel, M., Carter, B. D., Rees, D. E., \& Collier Cameron, A. 1997, MNRAS, 291, 658

Dupret, M.-A., Böhm, T., Goupil, M.-J., Catala, C., \& Grigahcene, A. 2006, Commun. Asteroseismol., 147, 72

Dupret, M.-A., Théado, S., Böhm, T., et al. 2007, Commun. Asteroseismol., 150, 59

Feigelson, E. D., Lawson, W. A., \& Garmire, G. P. 2003, ApJ, 599, 1207

Fuhr, J. R., \& Wiese, W. L. 2006, J. Phys. Chem. Ref. Data, 35, 1669

Gilliland, R. L. 1986, ApJ, 300, 339

Grady, C. A., Woodgate, B., Torres, C. A. O., et al. 2004, ApJ, 608, 809

Guimarães, M. M., Alencar, S. H. P., Corradi, W. J. B., \& Vieira, S. L. A. 2006, A\&A, 457, 581

Herbig, G. H. 1960, ApJS, 4, 337

Hu, J. Y., The, P. S., \& de Winter, D. 1989, A\&A, 208, 213

Hu, J. Y., Blondel, P. F. C., The, P. S., et al. 1991, A\&A, 248, 150

Iben, Jr., I. 1965, ApJ, 141, 993

Iben, I., \& Renzini, A. 1984, Phys. Rep., 105, 329

Kennelly, E. J. 1994, Ph.D. Thesis, The University Of British Columbia (Canada)

Kennelly, E. J., Walker, G. A. H., Matthews, J. M., \& Merryfield, W. J. 1993, in GONG 1992, Seismic Investigation of the Sun and Stars, ed. T. M. Brown, ASP Conf. Ser., 42, 359
Kennelly, E. J., Walker, G. A. H., Catala, C., et al. 1996, A\&A, 313, 571

Kochukhov, O. P. 2007, in Physics of Magnetic Stars, Proc. Int. Conf., Special Astrophysical Observatory of the Russian AS, 109

Kupka, F., Piskunov, N., Ryabchikova, T. A., Stempels, H. C., \& Weiss, W. W. 1999, A\&AS, 138, 119

Kupka, F. G., Ryabchikova, T. A., Piskunov, N. E., Stempels, H. C., \& Weiss, W. W. 2000, Baltic Astron., 9, 590

Kurtz, D. W., \& Marang, F. 1995, MNRAS, 276, 191

Kurtz, D. W., \& Müller, M. 1999, MNRAS, 310, 1071

Kuschnig, R., Weiss, W. W., Gruber, R., Bely, P. Y., \& Jenkner, H. 1997, A\&A, 328,544

Lenz, P., \& Breger, M. 2005, Commun. Asteroseismol., 146, 53

Lignieres, F., Catala, C., \& Mangeney, A. 1996, A\&A, 314, 465

Luhman, K. L. 2004, ApJ, 616, 1033

Lyo, A.-R., Lawson, W. A., \& Bessell, M. S. 2008, MNRAS, 389, 1461

Malkov, O. Y. 2007, MNRAS, 382, 1073

Marconi, M., \& Palla, F. 1998, ApJ, 507, L141

Marconi, M., \& Palla, F. 2004, in The A-Star Puzzle, ed. J. Zverko, J. Ziznovsky, S. J. Adelman, \& W. W. Weiss, IAU Symp., 224, 69

Meeus, G., Waters, L. B. F. M., Bouwman, J., et al. 2001, A\&A, 365, 476

Meléndez, J., \& Barbuy, B. 2009, A\&A, 497, 611

Palla, F., \& Stahler, S. W. 1990, ApJ, 360, L47

Palla, F., \& Stahler, S. W. 1993, ApJ, 418, 414

Palla, F., \& Stahler, S. W. 1999, ApJ, 525, 772

Palla, F., \& Stahler, S. W. 2001, ApJ, 553, 299

Piskunov, N. E., Kupka, F., Ryabchikova, T. A., Weiss, W. W., \& Jeffery, C. S. 1995, A\&AS, 112, 525

Praderie, F., Felenbok, P., Czarny, J., Talavera, A., \& Boesgaard, A. M. 1982, ApJ, 254, 658

Praderie, F., Catala, C., Simon, T., \& Boesgaard, A. M. 1986, ApJ, 303, 311

Press, W. H., Teukolsky, S. A., Vetterling, W. T., \& Flannery, B. P. 1992, Numerical recipes in FORTRAN, The art of scientific computing

Reegen, P. 2007, A\&A, 467, 1353

Ripepi, V., Marconi, M., Bernabei, S., et al. 2003, A\&A, 408, 1047

Ryabchikova, T. A., Piskunov, N. E., Kupka, F., \& Weiss, W. W. 1997, Baltic Astron., 6, 244

Siess, L., Dufour, E., \& Forestini, M. 2000, A\&A, 358, 593

Smalley, B. 2005, Mem. Soc. Astron. Ital. Suppl., 8, 130

Sneden, C. A. 1973, Ph.D. Thesis, The University of Texas at Austin

Sousa, S. G., Alapini, A., Israelian, G., \& Santos, N. C. 2010, A\&A, 512, A13

Testa, P., Huenemoerder, D. P., Schulz, N. S., \& Ishibashi, K. 2008, ApJ, 687, 579

van den Ancker, M. E., The, P. S., Tjin A Djie, H. R. E., et al. 1997, A\&A, 324, L33

van den Ancker, M. E., de Winter, D., \& Tjin A Djie, H. R. E. 1998, A\&A, 330, 145

Vick, M., Michaud, G., Richer, J., \& Richard, O. 2011, A\&A, 526, A37

Wade, G. A., Bagnulo, S., Drouin, D., Landstreet, J. D., \& Monin, D. 2007, MNRAS, 376, 1145

Waters, L. B. F. M., \& Waelkens, C. 1998, ARA\&A, 36, 233

Zima, W. 2008, Commun. Asteroseismol., 155, 17

Zima, W., Wright, D., Bentley, J., et al. 2006, A\&A, 455, 235

Zwintz, K. 2008, ApJ, 673, 1088

Zwintz, K., Marconi, M., Kallinger, T., \& Weiss, W. W. 2004, in The A-Star Puzzle, ed. J. Zverko, J. Ziznovsky, S. J. Adelman, \& W. W. Weiss, IAU Symp., 224, 353 